\title{
Right eye pain
}

\author{
Yu-Ming Weng • Hung-Jung Lin • Wei-Jing Lee
}

Received: 10 July 2009 /Accepted: 5 October 2009 / Published online: 19 November 2009

(C) Springer-Verlag London Ltd 2009

An 18-year-old man presented with severe right eyeball pain after an accidental injury via a compressed air tube. Severe eyeball pain along with tearing and photophobia were noted immediately after the compressed air injury. Ophthalmic examinations showed conjunctival injections (Fig. 1) without obvious corneal wound, foreign materials or increase in intraocular pressure (IOP). Orbital computed tomography (CT) was then arranged which showed subconjunctival emphysema (Fig. 2). No obvious orbital wall fracture or optic nerve entrapment were noted on any section of the orbital CT scan. He was discharged with topical medications and scheduled for outpatient follow-up.

Subconjunctival emphysema is a condition that is associated with subcutaneous emphysema due to laxity of the conjunctival tissues. Subconjunctival emphysema could be caused by high-pressure injury without obvious entry [1].

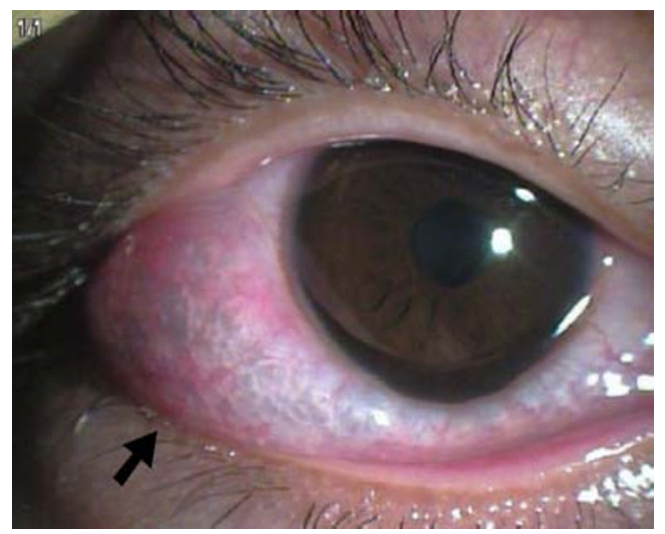

Fig. 1 External photograph of right eye shows lid oedema, conjunctival injections and subconjunctival emphysema (arrow)

Y.-M. Weng $\cdot$ H.-J. Lin $\cdot$ W.-J. Lee $(\triangle)$

Department of Emergency Medicine, Chi-Mei Medical Center, 901 Chung-Hua Road,

Yung-Kang City, Tainan 710, Taiwan e-mail: saab934151@pchome.com.tw

e-mail: echolee1103@gmail.com

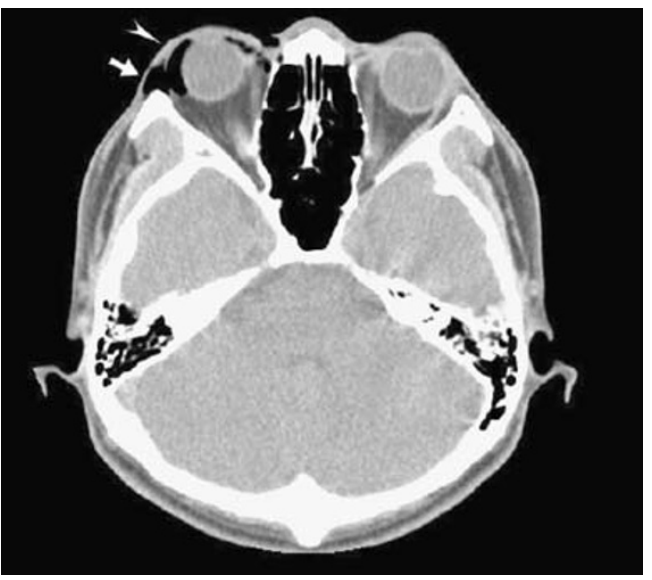

Fig. 2 Orbital emphysema (arrow) and subconjunctival emphysema (arrowhead)

Other combined injuries should also be considered including foreign body penetration, orbital wall fracture, eyeball structure injury, secondary infection, traumatic optic atrophy etc. Initial management usually involves conservative treatment with removal of the foreign body and bandaging of the eye with antibiotic ointment. Urgent emergency needle decompression is indicated when signs of decreased vision and increased IOP are present [2]. However, potential complications of needle decompression such as retrobulbar haemorrhage, sclera perforation and optic nerve damage could occur [2]. The patient should keep away from exposure to low atmospheric pressure such as during air travel to avoid tension pneumo-orbit.

\section{References}

1. Li T, Mafee MF, Edward DP (1999) Bilateral orbital emphysema from compressed air injury. Am J Ophthalmol 128(1):103-104

2. Singh M, Phua VM, Sundar G (2007) Sight-threatening orbital emphysema treated with needle decompression. Clin Experiment Ophthalmol 35:386-387 